\title{
Effect of Postnatal High-Protein Diet on Kidney Function of Rats Exposed to Intrauterine Protein Restriction
}

\author{
JING CHEN, HONG XU, QIAN SHEN, WEI GUO, AND LI SUN \\ Department of Nephrology, Children's Hospital, Fudan University, Shanghai 200032, People's Republic of China
}

\begin{abstract}
Poor fetal growth is linked with long-term detrimental effects on health in late life. We have previously shown that maternal protein restriction leads to hypertension and a reduced number of glomeruli in adult offspring. The aim of this study was to investigate the influence of a postnatal high-protein (HP) diet on renal development and renal function in rats subjected to a low-protein (LP) diet in fetal life. Sprague-Dawley rats were fed an LP diet throughout pregnancy. Male pups were given either a normal-protein (NP) diet (LP/NP) or HP diet (LP/HP), and normal male pups as control (NP/NP). At $12 \mathrm{wk}, \mathrm{LP} / \mathrm{HP}$ offspring displayed no increase in glomerular number but showed elevated blood pressure and proteinuria compared with the LP/NP group. There was minimal fusion of foot processes in LP/NP rats compared with a moderate fusion of foot processes and hyperplasia of mesangial cells in LP/HP rats. Renal desmin mRNA levels were elevated in both LP/NP and LP/HP groups but more significantly in the LP/HP group. This study suggests that postnatal HP diet amplifies the renal damage induced by fetal under-nutrition. Podocyte injury may be one of the mechanisms by which fetal protein restriction leads to proteinuria. (Pediatr Res 68: 100-104, 2010)
\end{abstract}

$\mathrm{E}^{\mathrm{p}}$ pidemiologic studies have suggested an inverse relationship between fetal growth and the risk for many adult diseases, including hypertension, type 2 diabetes mellitus, and chronic kidney disease (1-3). This suggests that factors in the prenatal and early postnatal environments can "program" an individual for an increased risk of certain diseases in later life. We have recently shown that maternal dietary protein restriction throughout pregnancy in rats, leads both to a decrease in glomerular number and to hypertension in adult offspring (4). The factors responsible for maximizing nephrogenic potential, and maintaining renal functional reserve, should be a major research priority.

It was believed that a postnatal high-protein (HP) diet is helpful for catch-up growth in children with intrauterine growth retardation (5). However, it has recently been proposed that a mismatch of the prenatal and postnatal environment can increase the risk of adult disease, i.e. the "predictive adaptive response" theory (6). In mice, merely giving ade-

Received September 9, 2009; accepted April 21, 2010.

Correspondence: Hong Xu, M.D., Ph.D, Children's Hospital of Fudan University, 399 Wan Yuan Road, Shanghai 201102, People's Republic of China; e-mail: hxu@shmu.edu.cn; cjcjcjs@163.com

Supported by grants from the National Natural Science Foundation (No.30672242) and the Young Science Foundation of Fudan University (No.08FQ42). quate postnatal nutrition reduces longevity in prenatally deprived offspring (7). High protein in itself may lead to glomerular hyperfusion and hyperfiltration, resulting in more serious damage to the kidney (8).

Based on these concerns, we have designed a study in which there is a disparity between the prenatal and postnatal environment; specifically, we have set out to investigate the influence of a postnatal HP diet on renal development and renal function in rats with maternal protein restriction.

\section{MATERIALS AND METHODS}

Animals and diets. The animal study protocols were approved by the Scientific and Ethics Committees at Children's Hospital of Fudan University. Virgin female Sprague-Dawley rats (250-300 g) were used in this experiment. They were mated and assumed to be pregnant when the vaginal plugs were expelled. One group of dams was maintained on a normal-protein diet (NP diet, 22\% protein) throughout pregnancy and lactation (NP/NP group). The other group of dams was placed on a low-protein diet (LP diet, 6\% protein), as described by Jones et al. (9), throughout gestation, and then switched to the NP diet (LP/NP group) or an HP diet $(30 \%$ protein, LP/HP group) on the day of delivery. The pups were weighed in the morning of the first day of postnatal life, no later than $15 \mathrm{~h}$ after delivery. The litter size was adjusted to eight pups per litter. All pups were nursed by their mothers until weaning and maintained on that diet until adulthood. The animals were fed ad libitum and housed in the same room with a constant temperature maintained at $25^{\circ} \mathrm{C}$ and a $12-\mathrm{h}$ light-dark cycle. To avoid possible gender differences, 10 male offspring of each group were studied in all phases of the project.

The three diets were isocaloric. The NP diet contained $22.2 \%$ protein, $3.31 \%$ fat, and $51.3 \%$ carbohydrate; the corresponding values for the other diets were $6.16 \%, 4.526 \%$, and $71.03 \%$ for LP and $30.10 \%, 3.16 \%$, and $42.42 \%$ for HP, respectively. The mineral and vitamin contents in the three diets were identical and were in accordance with the requirements for standard rat diets. The components of the NP diet contained $26 \%$ corn, $34 \%$ wheat, $2 \%$ alfalfa meal, $27 \%$ soybean meal, $5 \%$ fishmeal, $1 \%$ soybean oil, and $5 \%$ premix (mineral mixture, vitamins, etc). The components of the LP diet consisted of $50 \%$ corn, $13 \%$ saccharose, $26.5 \%$ starch, $2.5 \%$ soybean oil, $3 \%$ fishmeal, and $5 \%$ premix. The components of the HP diet contained $15 \%$ corn, $21 \%$ wheat flour, $51 \%$ soybean meal, $2 \%$ alfalfa meal, $2.5 \%$ casein, $2.5 \%$ fishmeal, $1 \%$ soybean oil, and $5 \%$ premix.

Experimental procedure and sample collection. At 4, 8, and 12 wk of age, 24-h urine collections were performed on the animals for the determination of protein excretion by the Coomassie Brilliant Blue G-250 method. Systolic blood pressures were measured oscillometrically by the tail-cuff method. The rats were trained to remain calm in a temperature-controlled Plexiglas restrainer during the measurements. The mean of four to six readings was recorded at each session.

The offspring were humanely euthanized using an overdose of sodium pentobarbital (i.p. injection) at $12 \mathrm{wk}$ of age. The left kidneys were removed immediately, washed with a $0.9 \% \mathrm{NaCl}$ solution to remove gross blood, weighed, and sectioned coronally. One half of the kidneys were fixed in

Abbreviations: HP, high-protein; LP, low-protein; NP, normal-protein 
formalin and embedded in paraffin for histologic analysis. Portions of the other half of the kidneys' cortexes were snap-frozen in liquid nitrogen and stored at $-80^{\circ} \mathrm{C}$ before analysis. A separate portion of the kidney cortical tissue was cut into $1-\mathrm{mm}^{3}$ pieces, fixed in $2.5 \%$ glutaraldehyde, and embedded in Araldite for electron microscopic analysis. The right kidneys were used for determining the number of glomeruli.

Determination of the number of nephrons. The total number of glomeruli per kidney was determined by the maceration method $(10,11)$. The right kidney was perfused with 5\% Alcian blue to stain the glomeruli. Each kidney was cut into small pieces in $1 \% \mathrm{NH}_{4} \mathrm{Cl}$ and then gently agitated in $50 \% \mathrm{HCl}$ for $2 \mathrm{~h}$ at room temperature. After mechanical dissociation, the homogenate was increased to a $10-\mathrm{mL}$ volume with distilled water. Twenty $20-\mu \mathrm{L}$ aliquots were pipetted onto concave slides for the estimation of glomeruli under a microscope. All determinations were performed by the same individual in a blinded fashion with an intra-individual variation of less than $15 \%$.

Histopathology evaluation. Sections (4- $\mu \mathrm{m}$ thickness, three to four per kidney) of paraffin-embedded tissue were stained with hematoxylin and eosin (HE) and Periodic Schiff reagent (PAS). The morphometric analysis was performed with a system composed of a microscope (BH2, Olympus, Japan) attached to a camera (MP-CP410, Panasonic, Japan) and a computer (M3120V, Ienovo, China), using image analysis software (IMS, China).

In each animal, the mean glomerular tuft volume $\left(V_{\mathrm{G}}\right)$ was determined from the mean glomerular cross-sectional area $\left(A_{\mathrm{G}}\right)$ by light microscopy, as previously described (12). The areas were estimated by light microscopy from an average area of 50 glomerular profiles (i.e. the capillary tuft omitting the proximal tubular tissue and the Bowmann capsule). The glomeruli that were counted were from the cortical area immediately under the capsular. $V_{\mathrm{G}}$ was calculated as $V_{\mathrm{G}}=$ $\left[(\beta / k)\left(A_{\mathrm{G}}\right)\right]^{3 / 2}$, where $\beta=1.38$ is the shape coefficient for spheres (the idealized shape of glomeruli) and $k=1.1$ is a size distribution coefficient.

The mesangial matrix area was quantified as described previously (13). The ratio of the PAS-positive area to the whole glomerular area was calculated to determine the mesangial matrix index (MMI). The mean of each glomerular MMI in the specimens was regarded as representing the magnitude of matrix accumulation in each case.

Measurement of podocyte foot processes. Standard methods were used to make electron microscopy tissue blocks as mentioned previously. Two tissue blocks were selected randomly and ultra thin sections of the blocks were cut and put on a copper grid. Every two grids were made from one block. The grids were stained with uranyl acetate and lead citrate and examined by transmission electron microscopy (H-7000, Hitachi, Japan). Micrographs from each grid were selected in a random fashion. An average of six micrographs per cross section and covering one fourth of the total cross section was photographed, giving a final magnification of 20000-fold. Only foot processes entirely on the peripheral glomerular basement membrane were measured (14).

Each photograph was analyzed with image analysis software (IMS, China). The apparent foot process width was classified using a log reciprocal scale. The number of podocyte foot processes along the glomerular basement membrane was counted by hand and the harmonic foot process width was calculated using the formula in the literature (15).

Desmin mRNA expression. Total RNA was isolated from snap-frozen kidneys using the TRIzol procedure (Invitrogen). The RNA was treated with DNase (Promega) and subjected to phenol-chloroform extraction and ethanol precipitation. The extracted RNA was measured by agarose gel electrophoresis for quality and by spectrometry for quantity. The $\mathrm{OD}_{260} / \mathrm{OD}_{280}$ ratios were within the range of $1.8-2.0$. The cDNA was synthesized from $1 \mu \mathrm{g}$ of total RNA using Moloney murine leukemia virus-reverse transcriptase (Promega).

Fluorescence real-time quantitative PCR was performed using an ABI PRISM 7000 sequence detection system (Applied Biosystems, CA). The reverse transcription reaction system had a total volume of $50 \mu \mathrm{L}$ and contained $25 \mu \mathrm{L}$ of SYBR Green Realtime PCR Master Mix (Toyobo, Japan), $1 \mu \mathrm{L}$ of each of the primers $(10 \mu \mathrm{M}), 2 \mu \mathrm{L}$ of cDNA and RNase-free $\mathrm{dH}_{2} \mathrm{O}$. Glyceraldehyde phosphate dehydrogenase (GAPDH) was used as a housekeeping gene for the analysis of the genes of interest. Desmin (NM022531): sense: 5'-TCAAGGGCACCAACGACT-3'; antisense: 5'-GGTCTGGATCGGAAGGTTGAT-3' (253 bp); GAPDH (NM017008): sense: 5'-CGGCAAGTTCAACGGCACAG-3'; antisense: 5'-ACGCCAGTAGACTCCACGAC-3' (144 bp). The thermal cycling conditions consisted of one cycle of $5 \mathrm{~min}$ at $95^{\circ} \mathrm{C} ; 40$ cycles of $30 \mathrm{~s}$ at $95^{\circ} \mathrm{C}, 30 \mathrm{~s}$ at $60^{\circ} \mathrm{C}\left(\mathrm{GAPDH}: 62^{\circ} \mathrm{C}\right)$, and $30 \mathrm{~s}$ at $72^{\circ} \mathrm{C}$; and subsequent melting curve analysis and cooling to $40^{\circ} \mathrm{C}$. The samples were analyzed in duplicate. The standard curve for the target gene was generated under the same condition, and the cycle threshold $(\mathrm{Ct})$ values of the samples were used to calculate the corresponding gene copy numbers. The relative quantities of mRNA are presented as the common logarithm of the copy numbers of the targeted gene/the copy numbers of GAPDH $\left(\times 10^{5}\right)$.

Statistical analyses. The results are expressed as means \pm SD. All data were entered and analyzed using the SPSS 10.0 statistical package. Differ- ences between groups were determined by one-way ANOVA, followed by LSD post hoc analysis. Statistical significance was defined as $p<0.05$.

\section{RESULTS}

Body and kidney weight. The birth weights of the pups from LP pregnancies were approximately 30\% lower than those from control pregnancies, which is consistent with our previous observations (4). The body weights remained low in the LP/NP group throughout life. In the LP/HP group, the body weights were lower than the controls at every time-point studied, although they were heavier than LP/NP rats at $4 \mathrm{wk}$ of age (Table 1).

At 12 wk of age, LP/NP offspring had significantly smaller kidneys than NP/NP offspring, but their kidney-to-body weight ratio was increased. The kidney weight was not significantly different in LP/HP groups when compared with controls at 12 wk. However, the kidney-to-body weight ratio was significantly higher in LP/HP animals than in the LP/NP and NP/NP groups (Table 1).

Number of nephrons. In adult LP/NP and LP/HP groups, the total glomerular number was significantly lower in both $\mathrm{LP} / \mathrm{NP}$ and LP/HP rats than in the NP/NP group (LP/NP, $\mathrm{LP} / \mathrm{HP}$ versus. NP/NP, $p<0.01$, respectively). There was no significant difference between the LP/NP and LP/HP rats (Table 2).

Blood pressure and urinary protein. Systolic blood pressure was measured at 4,8 , and $12 \mathrm{wk}$ of age. The LP/NP rats developed increased blood pressure levels by 8 wk of age, with further increases by $12 \mathrm{wk}$. The LP/HP group had the highest blood pressures, with the values significantly higher than those in the LP/NP group at 12 wk (Fig. 1).

Proteinuria in LP/NP and LP/HP rats at $12 \mathrm{wk}$ of age was significantly increased compared with the controls. The LP/HP rats had more severe proteinuria than the LP/NP group (Fig. 2).

Morphologic and morphometric analysis. The mean glomerular volume at $12 \mathrm{wk}$ was significantly increased in the LP/NP and LP/HP animals compared with the control group $\left(\mu \mathrm{m}^{3} \times 10^{5}\right)(\mathrm{LP} / \mathrm{NP}$ versus. NP/NP, $p<0.01 ; \mathrm{LP} / \mathrm{HP}$ versus. NP/NP, $p<0.001)$. The glomerular volume of the LP/HP group was greater than that in the LP/NP group $(p<0.001)$ (Fig. 3). The extracellular matrix area was also increased in the LP/HP group (LP/HP versus. NP/NP, $p<0.01$ ) (Fig. 4; Table 2).

Electron microscopy showed that the fusion of foot processes was marginal in the LP/NP rats, otherwise, there was obvious fusion of the foot processes and hyperplasia of mesangial cells in the LP/HP rats (Figs. 5 and 6). But quantitative analysis of foot processes revealed that there were no difference among the three groups (LP/NP, LP/HP versus. NP/NP; $271 \pm 64 \mathrm{~nm}, 277 \pm 66 \mathrm{~nm}$ versus. $264 \pm 46 \mathrm{~nm}, p>0.05$, respectively).

Desmin mRNA expression. Desmin is an early marker of podocyte damage (16). In our study, renal desmin mRNA levels, while increased in both LP/NP and LP/HP groups (LP/NP, LP/HP versus. NP/NP, $p<0.05$, respectively) showed the greatest increase in the LP/HP group (LP/HP versus. $\mathrm{LP} / \mathrm{NP}, p<0.05$ ) (Table 2 ). 
Table 1. Kidney and body weight

\begin{tabular}{lcccc}
\hline & Age $(\mathrm{wk})$ & NP/NP & LP/NP & LP/HP \\
\hline Body wt (g) & 4 & $87.4 \pm 5.6$ & $50.0 \pm 7.5^{*}$ & $57.6 \pm 7.2^{* \dagger}$ \\
& 8 & $282.2 \pm 49.1$ & $237.7 \pm 20.3 \ddagger$ & $239.5 \pm 53.3 \ddagger$ \\
Kidney wt (g) & 12 & $394.8 \pm 11.3$ & $300.0 \pm 26.9^{*}$ & $296.3 \pm 26.5^{*}$ \\
Kidney/body wt ratio (\%) & 12 & $1.320 \pm 0.061$ & $1.134 \pm 0.106 \ddagger$ & $1.316 \pm 0.204 \dagger$ \\
\hline
\end{tabular}

Data are mean $\pm \mathrm{SD} ; n=10$ /group.

$\ddagger p<0.05, * p<0.001, v s$. NP/NP; $\dagger p<0.05, \S p<0.001$, vs. value in LP/NP rats.

Table 2. Indices of glomerular geometry and Desmin mRNA expression

\begin{tabular}{|c|c|c|c|c|}
\hline Animal group & $\begin{array}{l}\text { Total number of glomeruli } \\
\text { per one kidney }\end{array}$ & $\begin{array}{l}\text { Mean glomerular volume } \\
\qquad\left(\mu \mathrm{m}^{3} \times 10^{5}\right)\end{array}$ & $\begin{array}{c}\text { Extracellular matrix } \\
\text { index }(\%)\end{array}$ & $\begin{array}{l}\text { Desmin mRNA } \\
\text { expression }\end{array}$ \\
\hline NP/NP & $28861 \pm 1044$ & $0.830 \pm 0.044$ & $21.3 \pm 2.1$ & $2.77 \pm 0.37$ \\
\hline LP/NP & $22900 \pm 926^{*}$ & $1.092 \pm 0.083^{*}$ & $23.2 \pm 1.8$ & $3.33 \pm 0.41 \dagger$ \\
\hline LP/HP & $23043 \pm 595^{*}$ & $1.508 \pm 0.097 \ddagger \S$ & $31.6 \pm 2.3^{*} \|$ & $4.06 \pm 0.27+\Phi$ \\
\hline
\end{tabular}

Data are mean $\pm \mathrm{SD} ; n=10 /$ group.

$\dagger p<0.05, * p<0.01, \ddagger p<0.001$, vs. NP/NP; II $p<0.05, \| p<0.01, \S p<0.001$, vs. value in LP/NP rats.

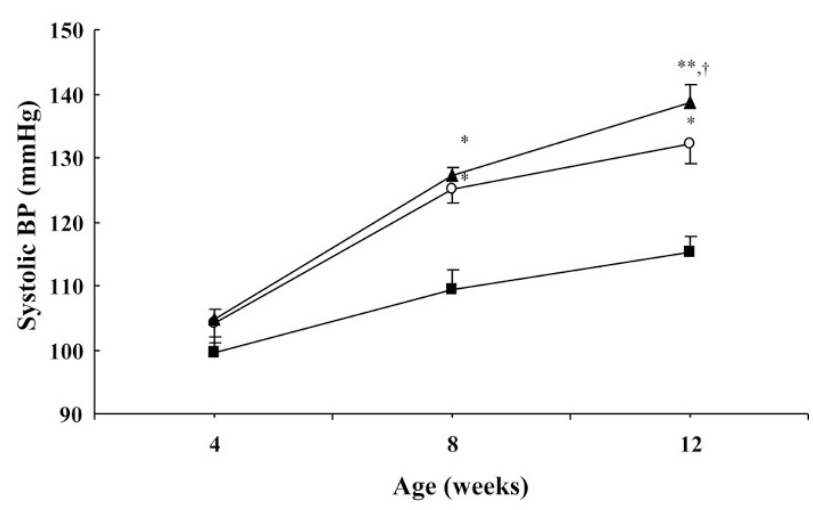

Figure 1. Systolic blood pressures in control offspring (NP/NP, $\square$ ) and LP offspring fed either a control (LP/NP, O) or an HP diet (LP/HP, $\mathbf{\Delta})$. Data are mean $\pm \mathrm{SD} ; n=10 /$ group; $* p<0.01,{ }^{* *} p<0.001$, vs. NP/NP; $\dagger p<0.01$, vs. value in LP/NP rats.

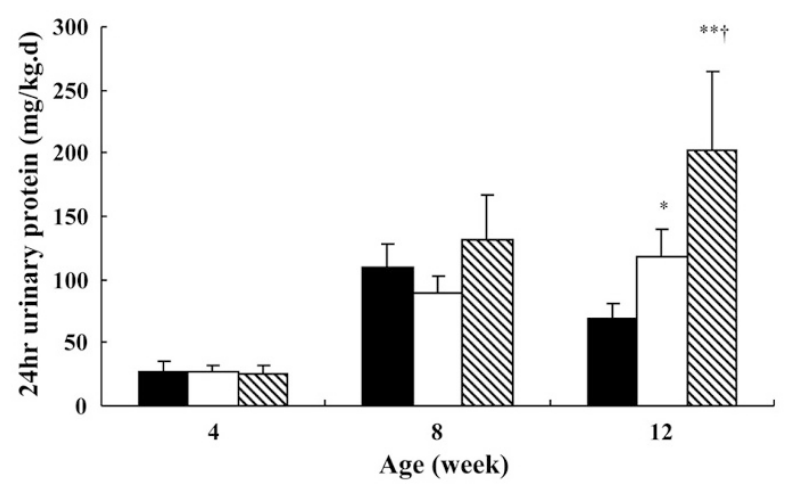

Figure 2. Twenty-four-hour urinary protein in control offspring (NP/NP, and LP offspring fed either a control (LP/NP, $\square$ ) or an HP diet (LP/HP, $\mathbb{Q})$. Data are mean $\pm \mathrm{SD} ; n=10 /$ group; $* p<0.05, * * p<0.001, v s . \mathrm{NP} / \mathrm{NP}$; $\dagger p<0.001, v s$. value in LP/NP rats.

\section{DISCUSSION}

Studies in both humans and experimental animals have established a relationship between an adverse intrauterine environment and offspring disease in adult life $(1,17)$. Re-
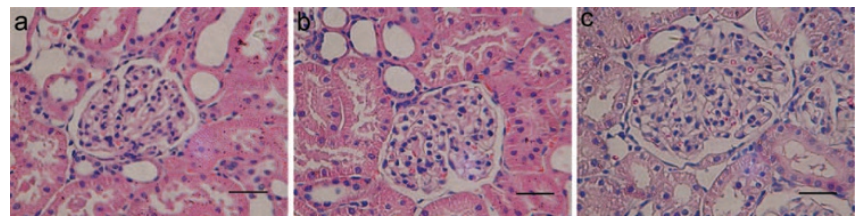

Figure 3. Photomicrographs of kidneys from 12 -wk old rats. Glomerular volume was significantly increased in LP/NP $(b)$ and LP/HP $(c)$ compared with NP/NP rats (a). In the LP/HP group, the glomerular volume was greater than in the LP/NP group. (HE $\times 250$, bar as $60 \mu \mathrm{m}$.)

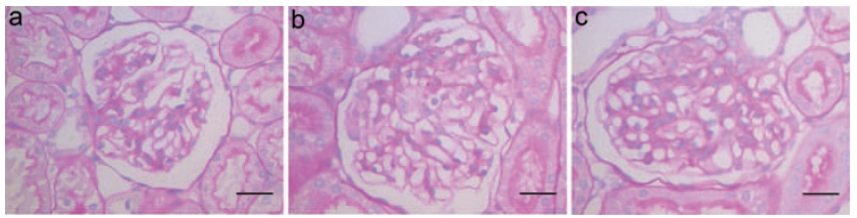

Figure 4. Photomicrographs of kidneys from 12-wk old rats. The glomerular extracellular matrix area was increased in LP/HP $(c)$ compared with that in NP/NP group $(a)$. There was no difference between LP/NP $(b)$ and NP/NP rats. (PAS $\times 400$, bar as $40 \mu \mathrm{m}$.)
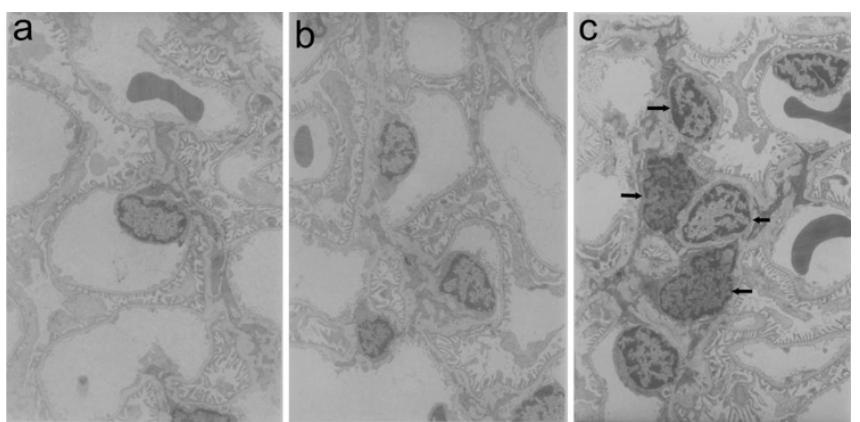

Figure 5. Electron microscopy showing hyperplasia of mesangial cells $(\uparrow)$ in LP/HP rats $(\times 2500)$. $(a, \mathrm{NP} / \mathrm{NP} ; b, \mathrm{LP} / \mathrm{NP} ; c, \mathrm{LP} / \mathrm{HP}$.

cently, however, it has been proposed that the mismatch between the prenatal and postnatal environment is an important determinant of subsequent disease, i.e. the "predictive adaptive response" theory (3). Our results confirm that a postnatal HP diet cannot rescue nephron deficits, but in fact 


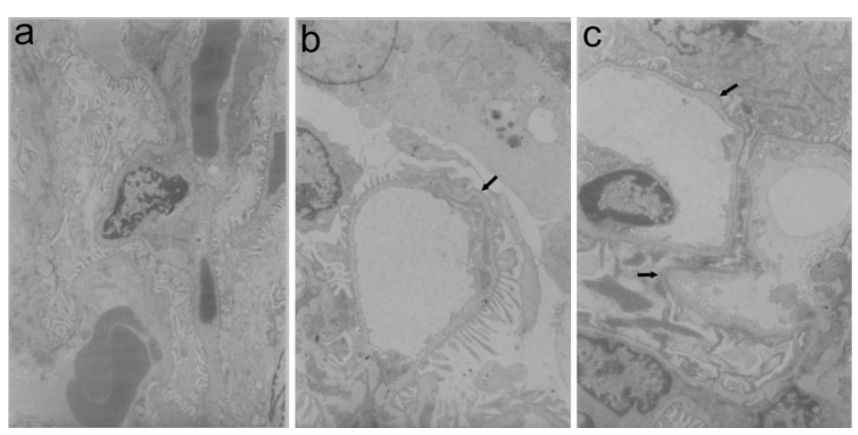

Figure 6. Electron microscopy showing occasional fusion of foot processes $(\uparrow)$ in LP/NP when compared with NP/NP rats, and much more evident fusion of foot processes $(\uparrow)$ in LP/HP rats $(\times 2500)$. ( $a$, NP/NP; $b$ LP/NP; $c$, LP/HP.)

amplifies the elevated blood pressure and proteinuria induced by fetal malnutrition.

About 15-30\% of IUGR children do not show catch-up growth after birth. Early postnatal growth is mainly regulated by nutrition, and Qiu et al. (18) proposed that an HP diet is an appropriate nutritional intervention in rats exposed to maternal under-nutrition to promote catch-up growth. In this study, both the LP/NP and LP/HP rats remained small until adulthood, even though there was a rapid weight gain in the LP/HP group at 4 wk of age. These were perhaps related to the more severe intervention of the LP diet throughout pregnancy.

In keeping with earlier observations, the LP/NP offspring developed worsening elevated blood pressure and mild proteinuria in later life (4). The total number of glomeruli was decreased by $\sim 21 \%$ in the LP/NP group. This reduced nephron number has been proposed as the common mechanism for various types of hypertension (19). Although we previously showed that the total renal GFR is not reduced in such pups (4), it is likely that single nephron hyperperfusion and hyperfiltration would be expected to partly compensate for the reduced nephron number. Our finding of increased glomerular volume and urinary protein excretion could be explained by single nephron hyperperfusion and hyperfiltration.

The offspring from LP mothers that were fed a postnatal HP diet showed no increase in nephron endowment, suggesting that the nephron deficit is determined before birth and is not compensated for after birth, even though nephrogenesis continues after birth. The postnatal HP diet amplified the renal abnormalities induced by fetal malnutrition; these included increased kidney-to-body weight ratio, glomerular volume expansion, elevated blood pressure and proteinuria. We also observed mesangial cell proliferation and extracellular matrix deposition in the LP/HP group. At present, the specific side effects of an HP diet have not been observed in healthy individuals. Lacroix et al. (20) reported that no major alterations of the kidneys were found in healthy rats consuming an HP diet (60\%) for 6 mo. HP during pregnancy did not lead to nephron endowment and hypertension in adulthood (21). In contrast, our results demonstrate that the LP offspring receiving a postnatal HP diet (30\%) for 12 wk show clear renal histologic lesions. We therefore propose that a postnatal HP diet may amplify the original glomerular hyperperfusion and hyperfiltration, resulting in more severe renal damage in adult rats subjected to a maternal LP diet.

The mechanism underlying the renal damage in the LP/NP and LP/HP groups remains to be determined. Although there was no statistically significant difference among the three groups in foot process width, it is worth noting that only minimal fusion of foot processes was present in LP/NP rats, compared with a partial fusion of foot processes in LP/HP rats. The foot process fusion is the most characteristic change in the podocyte structure response to injury. Thus, it is likely that podocyte injury is involved in the renal abnormalities induced by fetal programming. Podocyte damage was documented in our study by the expression of desmin, which is known to be a sensitive early marker of podocyte injury (16). In both LP/NP and LP/HP groups, renal desmin mRNA levels were greatly increased, particularly in the LP/HP group. Podocytes are highly sensitive to stretch and fluid shear stress $(22,23)$. Glomerular volume expansion and hyperfiltration, as seen in offspring from LP mothers, are likely to contribute to the structural damage of the podocyte. In particular, the LP/HP offspring showed both the highest desmin expression and partial fusion of the foot processes, suggesting that the postnatal environment, such as an HP diet, can amplify podocyte injury in programmed offspring. The amplification of the podocyte damage of fetal programming may be a result of increased mechanical stretch due to several processes, including greater volume expansion, mesangial cell proliferation, and extracellular matrix accumulation. We speculate that this might explain increased proteinuria.

In summary, this study in a model of a fetal LP diet confirms conclusions drawn from human epidemiologic studies that poor fetal growth is linked to long-term consequences on health in later life. However, we believe it is the first study to investigate the effects of a postnatal HP diet following intrauterine malnutrition on subsequent renal function. Our results show that a postnatal HP diet does not lead to increased glomerular number, but it does lead to more severe elevated blood pressure and proteinuria. The amplification of renal abnormalities by an HP diet seen in these growth-retarded animals suggests that postnatal environmental factors are important in the etiology of adult-onset disease. The findings in this study also suggest that damage to the podocyte may be one of the mechanisms by which fetal protein restriction and a postnatal HP diet lead to proteinuria.

Acknowledgment. We thank Mr. Leiwei Zhu for technical assistance in the kidney sampling process.

\section{REFERENCES}

1. Godfrey KM, Barker DJ 2000 Fetal nutrition and adult disease. Am J Clin Nutr 71:1344S-1352S

2. Painter RC, Roseboom TJ, Montfrans GA, Bossuyt PM, Krediet RT, Osmond C, Barker DJ, Bleker OP 2005 Microalbuminuria in adults after prenatal exposure to the Dutch famine. J Am Soc Nephrol 16:189-194

3. Gillman MW 2005 Developmental origins of health and disease. N Engl J Med 353:1848-1850

4. Chen J, Xu H, Shen Q, Guo W, Sun L, Ling SY 2006 Influence of IUGR on blood pressure and renal function in rats. Chin J Nephrol 22:706-707

5. Fewtrell MS, Morley R, Abbott RA, Singhal A, Stephenson T, MacFadyen UM, Clements H, Lucas A 2001 Catch-up growth in small-for- gestational-age term infants: a randomized trail. Am J Clin Nutr 74:516-523 
6. Gluckman PD, Hanson MA 2004 Living with the past: evolution, development and patterns of disease. Science 305:1733-1736

7. Ozanne SE, Hales CN 2004 Catch-up growth and obesity in male mice. Nature 427:411-412

8. Lentine K, Wrone EM 2004 New insights into protein intake and progression of renal disease. Curr Opin Nephrol Hypertens 13:333-336

9. Jones SE, Bilous RW, Flyvbjerg A, Marshall SM 2001 Intra-uterine environment influences glomerular number and acute renal adaptation to experimental diabetes. Diabetologia 44:721-728

10. Bankir L, Hollenberg NK 1983 In vivo staining of the kidney with Alcian blue: an adjunct to morphological and physiological studies. Ren Physiol 6:151-155

11. Martins JP, Monteiro JC, Paixao AD 2003 Renal function in adult rats subjected to prenatal dexamethasone. Clin Exp Pharmacol Physiol 30:32-37

12. Schrijvers BF, Rasch R, Tilton RG, Flyvbjerg A 2002 High protein-induced glomerular hypertrophy is vascular endothelial growth factor-dependent. Kidney Int $61: 1600-1604$

13. Kuriki M, Asahi K, Asano K, Sakurai K, Eiro M, Suzuki H, Watanabe K, Katoh T, Watanabe T 2003 Steroid therapy reduces mesangial matrix accumulation in advanced IgA nephropathy. Nephrol Dial Transplant 18:1311-1315

14. Nevins TE, Gaston T, Basgen JM 1984 Quantitative indexes of aminonucleosideinduced nephrotic syndrome. Am J Pathol 117:30-36

15. Gundersen HJ, Seefeldt T, Osterby R 1980 Glomerular epithelial foot processes in normal man and rats. Distribution of true width and its intra- and inter-individual variation. Cell Tissue Res 205:147-155
16. Gross ML, Koch A, Muhlbauer B, Adamczak M, Ziebart H, Drescher K, Gross G, Berger I, Amann KU, Ritz E 2006 Renoprotective effect of a dopamine D3 receptor antagonist in experimental type II diabetes. Lab Invest 86:262-274

17. Tulassay T, Vasarhelyi B 2002 Birth weight and renal function. Curr Opin Nephrol Hypertens 11:347-352

18. Qiu XS, Huang TT, Shen ZY, Deng HY, Ke ZY, Mei KY, Lai F 2004 Effect of different early nutritional interventions on catch-up growth of rats with intrauterine growth retardation. Zhonghua Er Ke Za Zhi 42:782-786

19. Brenner BM, Chertow GM 1994 Congenital oligonephropathy and the etiology of adult hypertension and progressive renal injury. Am J Kidney Dis 23:171-175

20. Lacroix M, Gaudichon C, Martin A, Morens C, Mathe V, Tome D, Huneau JF 2004 A long-term high-protein diet markedly reduces adipose tissue without major side effects in Wistar male rats. Am J Physiol Regul Integr Comp Physiol 287:R934R942

21. Zimanyi MA, Bertram JF, Black MJ 2002 Nephron number and blood pressure in rat offspring with maternal high-protein diet. Pediatr Nephrol 17:1000-1004

22. Dalla Vestra M, Masiero A, Roiter AM, Saller A, Crepaldi G, Fioretto P 2003 Is podocyte injury relevant in diabetic nephropathy? Studies in patients with type 2 diabetes. Diabetes 52:1031-1035

23. Friedrich C, Endlich N, Kriz W, Endlich K 2006 Podocytes are sensitive to fluid shear stress in vitro. Am J Physiol Renal Physiol 291:F856-F865 